\title{
Quantum Moment Map and Invariant Integration Theory on Quantum Spaces
}

\author{
O. Osuna Castro, E. Wagner
}

\begin{abstract}
It is shown that, on the one hand, quantum moment maps give rise to examples for the operator-theoretic approach to invariant integration theory developed by K.-D. Kürsten and the second author, and that, on the other hand, the operator-theoretic approach to invariant integration theory is more general since it also applies to examples without a well-defined quantum moment map.
\end{abstract}

Keywords: quantum spaces, invariant integration theory, quantum moment map.

\section{Introduction}

A noncommutative analogue of an (infinitesimal) group action on a topological space is described by the action of a Hopf algebra on a noncommutative function algebra. In this setting, a generalization of the classical Haar measure is given by an invariant integral, that is, a positive linear functional with certain invariance properties. Usually the noncommutative function algebra is generated by a finite set of generators which are considered as coordinate functions on the quantum space. As in the classical case, one does not expect that polynomials in the coordinate functions on locally compact quantum spaces are integrable. This leads to the problem that one has to associate algebras of integrable (and differentiable) functions to the noncommutative polynomial algebra in an appropriate way. In the algebraic approach (see e.g. [2, 7]), one associates function algebras by imposing commutation relations with the generators and defines the invariant integral by Jackson-type integrals. A more rigorous method was developed by Kürsten and the second author in [3], based on Hilbert space representations and (unbounded) operator algebras. The advantage of this method becomes apparent in the examples of [5], where the algebraic approach would fail.

The first step of the operator-theoretic approach is to express the action of a Hopf $*$-algebra $\mathcal{U}$ on a *-algebra $\mathcal{A}$ by algebraic relations of Hilbert space operators. It should be noted that the operators describing the action do not have to satisfy the commutation relations of $\mathcal{U}$. On the other hand, any joint representation of $\mathcal{U}$ and $\mathcal{A}$ on the same Hilbert space allows one to equip $\mathcal{A}$ with a $\mathcal{U}$-action, given by the formulas of the adjoint action, provided that $\mathcal{A}$ is invariant under these algebraic expressions [6]. This will be automatically the case if there is a *-homomorphism from $\mathcal{U}$ into $\mathcal{A}$. Then one only has to consider ${ }^{*}$-representations of $\mathcal{A}$ on a Hilbert space and the methods from [3] will apply without restrictions. In [2], Korogodsky called a *-homomorphism from $\mathcal{U}$ into $\mathcal{A}$ intertwining the (adjoint) action a "quantum moment map".

The aim of this paper is to show that, on the one hand, quantum moment maps give rise to examples for the operator-theoretic approach to invariant integration theory. On the other hand, we demonstrate that the operator-theoretic approach to invariant integration theory is more general, since it also applies to cases where the operators describing the action do not satisfy the commutation relations of $\mathcal{U}$ and hence do not define a quantum moment map.

\section{Operator-theoretic approach to invariant integration theory}

For details on quantum groups and related notions, we refer the reader to [1]. Let $\mathcal{U}$ be a Hopf *-algebra with Hopf structure $\Delta, \varepsilon$ and $S$, where $\Delta: \mathcal{U} \rightarrow \mathcal{U} \otimes \mathcal{U}$ and $\varepsilon: \mathcal{U} \rightarrow \mathbb{C}$ are ${ }^{*}$-homomorphisms and $S: \mathcal{U} \rightarrow \mathcal{U}$ is an anti-homomorphism satisfying certain conditions. We will use Sweedler-Heinemann notation and write $\Delta(f)=f_{(1)} \otimes f_{(2)}$.

A *-algebra $\mathcal{X}$ is called a left $\mathcal{U}$-module *algebra if there is a left $\mathcal{U}$-action $\triangleright$ on $\mathcal{X}$ such that

$$
\begin{aligned}
f \triangleright(x y) & =\left(f_{(1)} \triangleright x\right)\left(f_{(2)} \triangleright y\right), \\
(f \triangleright x)^{*} & =S(f)^{*} \triangleright x^{*}, \\
x, y & \in \mathcal{X}, \quad f \in \mathcal{U} .
\end{aligned}
$$

For unital algebras, one also requires $f \triangleright 1=\varepsilon(f) 1$. By an invariant integral we mean a positive linear functional $h$ on $\mathcal{X}$ satisfying

$$
h(f \triangleright x)=\varepsilon(f) h(x), \quad x \in \mathcal{X}, f \in \mathcal{U} .
$$


Given a dense linear subspace $D$ of a Hilbert space $\mathcal{H}$, consider the ${ }^{*}$-algebra

$$
\mathcal{L}^{+}(d):=\left\{x \in \operatorname{End}(D) ; D \subset D\left(x^{*}\right), x^{*} D \subset D\right\}
$$

with involution $x \mapsto x^{*} \uparrow_{D}$. An (unbounded) *-representation of $\mathcal{X}$ is a ${ }^{*}$-homomorphism $\pi: \mathcal{X} \rightarrow$ $\mathcal{L}^{+}(d)$. If for each $f \in \mathcal{U}$ there exists a finite number of operators $L_{i}, R_{i} \in \mathcal{L}^{+}(d)$ such that

$$
\pi(f \triangleright x)=\sum_{i} L_{i} \pi(x) R_{i}, \quad x \in \mathcal{X}
$$

then we say that we have an operator expansion of the action. Obviously, it suffices to know the operators $L_{i}, R_{i}$ for a set of generators of $\mathcal{U}$.

Let $\mathfrak{A}$ denote the ${ }^{*}$-subalgebra of $\mathcal{L}^{+}(d)$ generated by $\pi(\mathcal{X})$ and the operators $L_{i}, R_{i}$ for a set of generators of $\mathcal{U}$. Set

$$
\begin{aligned}
\mathcal{S}(\mathfrak{A}):= & \left\{t \in \mathcal{L}^{+}(d) ; \bar{t} \mathcal{H} \subset D, \bar{t}^{*} \mathcal{H} \subset D,\right. \\
& \left.\overline{a t b} \in \mathcal{L}^{1}(\mathcal{H}) \quad \forall a, b \in \mathfrak{A}\right\},
\end{aligned}
$$

where the bar denotes the closure of closeable operators on $D$, and $\mathcal{L}^{1}(\mathcal{H})$ is the Schatten class of trace class operators on $\mathcal{H}$.

The *-algebra $\mathcal{S}(\mathfrak{A})$ will be considered as an algebra of differentiable functions which vanish sufficiently rapidly at "infinity". If the operators from the operator expansion satisfy convenient commutation relations (but not necessarily the defining relations of $\mathcal{U})$, then the $\mathcal{U}$-action can be expanded to $\mathcal{S}(\mathfrak{A})$. In favorable cases, one can define an invariant integral by a weighted trace on $\mathcal{S}(\mathfrak{A})$, where the weight is easily guessed from the operator expansion of the action by analogy to the well-known quantum trace (see $[3,5])$.

A Hopf algebra $\mathcal{U}$ acts always on itself by the (left) adjoint action:

$$
\operatorname{ad}_{\mathrm{L}}(f)(x):=f_{(1)} x S\left(f_{(2)}\right), \quad f, x \in \mathcal{U},
$$

In [2], L. I. Korogodsky defined a quantum moment map as a ${ }^{*}$-homomorphism $\rho: \mathcal{U} \rightarrow \mathcal{X}$ such that $\rho\left(\operatorname{ad}_{\mathrm{L}}(f)(x)\right)=f \triangleright \rho(x)$ for all $f, x \in \mathcal{U}$. Then any *-representation $\pi: \mathcal{X} \rightarrow \mathcal{L}^{+}(D)$ leads to a *-representation $\pi \circ \rho: \mathcal{U} \rightarrow \mathcal{L}^{+}(D)$, and it follows easily from the Hopf algebra structure of $\mathcal{U}$ that

$$
\begin{aligned}
\operatorname{ad}_{\mathrm{L}}(f)(X) & :=\pi\left(\rho\left(f_{(1)}\right)\right) X \pi\left(\rho\left(S\left(f_{(2)}\right)\right)\right), \\
f & \in \mathcal{U}, \quad X \in \mathcal{L}^{+}(D)
\end{aligned}
$$

defines a left $\mathcal{U}$-action on $\mathcal{L}^{+}(D)$ turning it into a $\mathcal{U}$ module *-algebra. Moreover, the algebra $\mathcal{S}(\mathfrak{A})$ is invariant under this action. Suppose furthermore that $\mathcal{U}$ denotes the Quantized Universal Enveloping Algebra of a semisimple Lie algebra. Then there exists a distinguished element $\Gamma$ in $\mathcal{U}$ such that $\Gamma f=S^{2}(f) \Gamma$ for all $f \in \mathcal{U}$. By the definition of $\mathcal{S}(\mathfrak{A})$, the traces
$\operatorname{tr}(\overline{\pi(\rho(\Gamma)) f})$ are well-defined and we can state the following theorem:

Theorem 1 Let $\rho: \mathcal{U} \rightarrow \mathcal{X}$ be a quantum moment map and $\pi: \mathcal{X} \rightarrow \mathcal{L}^{+}(D) a{ }^{*}$-representation such that $\pm \overline{\pi(\rho(\Gamma))}$ is a non-negative selfadjoint operator. Then

$$
h(f):= \pm \operatorname{tr}(\overline{\pi(\rho(\Gamma)) f}), \quad f \in \mathcal{S}(\mathfrak{A}),
$$

defines an invariant integral on the $\mathcal{U}$-module *-algebra $\mathcal{S}(\mathfrak{A})$.

Proof. The invariance of $h$ follows from the same formulas as in the proof of the invariance of the quantum trace in $[1$, Section 7.1.6] by applying the trace property $\operatorname{tr}(\overline{a f})=\operatorname{tr}(\overline{f a})$ which continues to hold for all $f \in \mathcal{S}(\mathfrak{A})$ and $a \in \mathfrak{A}$, see [3].

\section{Example: A quantum hyperboloid}

Let $q \in(0,1)$ and $s \in[-1,1)$. Following [2], we define the two-sheet quantum hyperboloid $\mathcal{X}:=\mathcal{O}_{q}\left(X_{s, 1}\right)$ (after a slight reparametrization) as the *-algebra generated by $y, y^{*}$ and $x=x^{*}$ with commutation relations

$$
\begin{aligned}
y x & =q^{2} x y, \quad x y^{*}=q^{2} y^{*} x, \\
y^{*} y & =\left(q^{-2} x-s\right)\left(q^{-2} x-1\right), \\
y y^{*} & =(x-s)(x-1) .
\end{aligned}
$$

The Hopf $*_{\text {-algebra } \mathcal{U}}:=\mathcal{U}_{q}\left(\mathrm{su}_{1,1}\right)$ is generated by $E, F, K$ and its inverse $K^{-1}$ with relations

$$
\begin{aligned}
K E & =q^{2} E K, \quad F K=q^{2} K F, \\
E F-F E & =\left(q-q^{-1}\right)^{-1}\left(K-K^{-1}\right),
\end{aligned}
$$

with Hopf structure

$$
\begin{aligned}
\Delta(E) & =E \otimes 1+K \otimes E, \\
\Delta(F) & =F \otimes K^{-1}+1 \otimes F, \\
\Delta(K) & =K \otimes K, \\
\varepsilon(E) & =\varepsilon(F)=0, \\
\varepsilon(K) & =1, \quad S(E)=-K^{-1} E, \\
S(F) & =-F K, \quad S(K)=K^{-1},
\end{aligned}
$$

and with involution $K^{*}=K, E^{*}=-K F$. The quantum hyperboloid $\mathcal{X}$ becomes a $\mathcal{U}$-module *-algebra with the action defined by

$$
\begin{aligned}
& K \triangleright y=q^{2} y, \quad E \triangleright y=0, \\
& F \triangleright y=q^{1 / 2}\left(\left(1+q^{-2}\right) x-(1+s)\right), \\
& K \triangleright x=x, \quad E \triangleright x=q^{1 / 2} y, \quad F \triangleright x=q^{5 / 2} y^{*}, \\
& K \triangleright y^{*}=q^{-2} y^{*}, \\
& E \triangleright y^{*}=q^{-3 / 2}\left(\left(1+q^{-2}\right) x-(1+s)\right), \quad F \triangleright y^{*}=0 .
\end{aligned}
$$


Let $I$ be an at most countable index set, $\mathcal{H}_{0}$ a Hilbert space, and $\mathcal{H}=\underset{i \in I}{\oplus} \mathcal{H}_{0}$. We denote by $\eta_{i}$ the vector of $\mathcal{H}$ which has the element $\eta \in \mathcal{H}_{0}$ as its $i$-th component and zero otherwise. It is understood that $\eta_{i}=0$ whenever $i \notin I$. Let $U$ be a unitary operator on $\mathcal{H}_{0}$, and let $A$ and $B$ be selfadjoint operators on the Hilbert space $\mathcal{H}_{0}$ such that $\operatorname{spec}(A) \subset\left[q^{2}, 1\right], \operatorname{spec}(B) \subset\left[q^{2}, s\right], q^{2}$ is not an eigenvalue of $A$ and $B$, and $s$ is not an eigenvalue of $B$. Set $\lambda_{n}:=\sqrt{\left(q^{2 n}-s\right)\left(q^{2 n}-1\right)}$ and $\lambda_{n}(t):=$ $\sqrt{\left(q^{2 n} t-s\right)\left(q^{2 n} t-1\right)}$. Then a list of non-equivalent *-representations of $\mathcal{X}$ is given by the following formulas (suppressing the letter $\pi$ of the representation).

$$
\begin{array}{ll}
s \in[-1,1): \quad & x \eta_{n}=q^{-2 n} \eta_{n}, \quad y \eta_{n}=\lambda_{-(n+1)} \eta_{n+1} \\
& \text { on } \mathcal{H}=\oplus_{n \in \mathbb{N}_{0}} \mathcal{H}_{0} . \\
s \in[0,1): \quad & x \eta_{n}=-q^{2(n+1)} A \eta_{n}, \quad y \eta_{n}=\lambda_{n}(-A) \eta_{n-1} \\
& \text { on } \mathcal{H}=\oplus_{n \in \mathbb{Z}} \mathcal{H}_{0} . \\
s \in(0,1): \quad & x \eta_{n}=q^{2(n+1)} s \eta_{n}, \quad y \eta_{n}=\lambda_{n}(s) \eta_{n-1} \\
& \text { on } \mathcal{H}=\oplus_{n \in \mathbb{N}_{0}} \mathcal{H}_{0} ; \\
& x=0, y=s U \text { on } \mathcal{H}_{0} . \\
s \in\left(q^{2}, 1\right): \quad & x \eta_{n}=q^{-2 n} s \eta_{n}, y \eta_{n}=\lambda_{-(n+1)}(s) \eta_{n+1} \\
& \text { on } \mathcal{H}=\oplus_{n \in \mathbb{N}_{0}} \mathcal{H}_{0} ; \\
& x \eta_{n}=q^{2(n+1)} \eta_{n}, y \eta_{n}=\lambda_{n} \eta_{n-1} \\
& \text { on } \mathcal{H}=\oplus_{n \in \mathbb{N}_{0}} \mathcal{H}_{0} ; \\
& x \eta_{n}=q^{2(n+1)} B \eta_{n}, y \eta_{n}=\lambda_{n}(B) \eta_{n-1} \\
& \text { on } \mathcal{H}=\oplus_{n \in \mathbb{Z}} \mathcal{H}_{0} . \\
& x=q^{2}, y=0 \text { on } \mathcal{H}_{0} . \\
s=q^{2}: & x=y=0 \text { on } \mathcal{H}_{0} . \\
s=0: & x \eta_{n}=q^{-2 n} s \eta_{n}, y \eta_{n}=\lambda_{-(n+1)}(s) \eta_{n+1} \\
s \in[-1,0): & \text { on } \mathcal{H}=\oplus_{n \in \mathbb{N}_{0}} \mathcal{H}_{0} .
\end{array}
$$

The domain $D$ of the representation can be chosen, for instance, to be the linear span of the $\eta_{n}$ 's. If one imposes some well-behavedness conditions, for instance that $\bar{x}$ is self-adjoint and that $y f(\bar{x}) \subset f(\bar{x}) y$ for all bounded measurable functions (with respect to the spectral measure of $\bar{x}$ ), then this list is complete in the sense that each well-behaved representation is a direct sum of representations from the above list. A single representation is irreducible if and only if $\mathcal{H}_{0}=\mathbb{C}$. In this case $A, B$ and $U$ become complex numbers such that $A \in\left(q^{2}, 1\right], B \in\left(q^{2}, s\right)$ and $|U|=1$. For the proof of these claims, see [4].

Given a ${ }^{*}$-representation such that $x$ is invertible in $\mathcal{L}^{+}(D)$, set

$$
\begin{aligned}
& e:=q^{-1 / 2}\left(q-q^{-1}\right)^{-1} x^{-1} y \\
& f:=-q^{1 / 2}\left(q-q^{-1}\right)^{-1} y^{*}, \quad k:=q x^{-1} .
\end{aligned}
$$

Direct computations show that

$$
\begin{aligned}
K \triangleright z & =k z k^{-1}, \quad E \triangleright z=e z-k z k^{-1} e, \\
F \triangleright z & =f z k-z f k
\end{aligned}
$$

for $z=x, y, y^{*}$. Using the relations

$$
\begin{aligned}
k e & =q^{2} e k, \quad f k=q^{2} k f, \\
e f-f e & =\left(q-q^{-1}\right)^{-1}\left(s k-k^{-1}\right),
\end{aligned}
$$

one easily proves that $(8)$ defines a $\mathcal{U}$-action on $\mathcal{L}^{+}(D)$ turning it into a left $\mathcal{U}$-module ${ }^{*}$-algebra. With $\mathfrak{A}$ being the ${ }^{*}$-subalgebra of $\mathcal{L}^{+}(D)$ generated by $y, y^{*}, x$ and $x^{-1}$, the ${ }^{*}$-algebra $\mathcal{S}(\mathfrak{A})$ defined in (4) becomes a left $\mathcal{U}$-module ${ }^{*}$-subalgebra of $\mathcal{L}^{+}(D)$. Since the traces of elements from $\mathcal{S}(\mathfrak{A})$ are welldefined, we can state the following proposition:

Proposition 2 If $\pm \bar{x}$ is a non-negative selfadjoint operator, then

$$
h(f):= \pm \operatorname{tr}\left(\overline{k^{-1} f}\right)
$$

defines an invariant integral on $\mathcal{S}(\mathfrak{A})$.

Proof. The invariance follows from the trace property $\operatorname{tr}(\overline{a f})=\operatorname{tr}(\overline{f a})$ for all $f \in \mathcal{S}(\mathfrak{A})$ and $a \in \mathfrak{A}$. As an example, we show the invariance with respect to $E$,

$$
\begin{aligned}
h(E \triangleright z)= & \pm \operatorname{tr}\left(\overline{k^{-1} e z-z k^{-1} e}\right)= \\
& \pm \operatorname{tr}\left(\overline{k^{-1} e z-k^{-1} e z}\right)=0=\varepsilon(E) h(z) .
\end{aligned}
$$

The positivity of $h$ is clear by the positivity of $\pm k^{-1}= \pm q^{-1} x$.

Note that Equation (8) is invariant under the rescaling $k \mapsto t k$ and $f \mapsto t^{-1} f$. If $t \in \mathbb{R} \backslash\{0\}$, the rescaling does not affect the involution, i.e., we have $k^{*}=k$ and $e^{*}=-k f$. From (9), it follows that

$$
\rho(K)=s^{1 / 2} k, \quad \rho(E)=e, \quad \rho(F)=s^{-1 / 2} f
$$

defines a moment map $\rho: \mathcal{U} \rightarrow \mathfrak{A}$ if and only if $s \in(0,1)$. In this situation, Proposition 2 is an immediate consequence of Theorem 1 together with the formula of the quantum trace.

However, we emphasize that Proposition 2 holds for all $s \in[-1,0)$, even if the operators $k, e$ and $f$ do not satisfy the defining relations of $\mathcal{U}_{q}\left(\mathrm{su}_{1,1}\right)$. This shows that the operator-theoretic approach to invariant integration theory is more general than the method based on a quantum moment map. We also would like to point out that our approach works for all representations from the above list where $x \neq 0$, even for those where $\bar{x}$ has a continuous spectrum, whereas in the algebraic approach, one usually considers functions in $x$ which are supported on a discrete set $[2,7]$.

\section{References}

[1] Klimyk, A. U., Schmüdgen, K.: Quantum Groups and Their Representations. Berlin : SpringerVerlag, 1997. 
[2] Korogodsky, L. I.: Representation of quantum algebras arising from non-compact quantum groups: quantum orbit method and super-tensor products, Ph.D. Thesis, Massachusetts Institute of Technology, Dept. of Math., 1996. Complimentary series representations and quantum orbit method. ArXiv:q-alg/9708026v1.

[3] Kürsten, K.-D., Wagner, E.: An operatortheoretic approach to invariant integrals on quantum homogeneous $\mathrm{SU}_{n, 1}$-spaces. Publ. Res. Inst. Math. Sci. 43 (1), 2007, p. 1-37.

[4] Lucio Peña, P. C., Osuna Castro, O., Wagner, E.: Invariant integration theory on the quantum hyperboloid, in preparation.

[5] Osuna Castro, O., Wagner, E.: An operatortheoretic approach to invariant integrals on quantum homogeneous $\mathrm{sl}(n+1, \mathbb{R})$-spaces. ArXiv: math.QA/0904.0669v1.
[6] Schmüdgen, K., Wagner, E.: Hilbert space representations of cross product algebras. J. Funct. Anal. 200 (2), 2003, p. 451-493.

[7] Shklyarov, D. L., Sinel'shchikov, S. D., Vaksman, L. L.: Integral representations of functions in the quantum disk. I. (Russian) Mat. Fiz. Anal. Geom. 4 (3), 1997, p. 286-308. Quantum Matrix Balls: Differential and Integral Calculi. ArXiv:math.QA/9905035.

Osvaldo Osuna Castro

E-mail: osvaldo@ifm.umich.mx

Department of Physics and Mathematics University of Michoacan

Morelia, Mexico

Elmar Wagner

E-mail: elmar@ifm.umich.mx

Department of Physics and Mathematics University of Michoacan

Morelia, Mexico 